\title{
Fast Decoding and Optimal Decoding for Machine Translation
}

\author{
Ulrich Germann $\dagger$, Michael Jahr $\ddagger$, Kevin Knight $\dagger$, Daniel Marcu $\dagger$, and Kenji Yamada $\dagger$ \\ $\dagger$ Information Sciences Institute \\ University of Southern California \\ 4676 Admiralty Way, Suite 1001 \\ Marina del Rey, CA 90292 \\ $\ddagger$ Department of Computer Science \\ Stanford University \\ Stanford, CA 94305 \\ jahrecs.stanford.edu
}

\{germann, knight, marcu, kyamada\}eisi.edu

\begin{abstract}
A good decoding algorithm is critical to the success of any statistical machine translation system. The decoder's job is to find the translation that is most likely according to set of previously learned parameters (and a formula for combining them). Since the space of possible translations is extremely large, typical decoding algorithms are only able to examine a portion of it, thus risking to miss good solutions. In this paper, we compare the speed and output quality of a traditional stack-based decoding algorithm with two new decoders: a fast greedy decoder and a slow but optimal decoder that treats decoding as an integer-programming optimization problem.
\end{abstract}

\section{Introduction}

A statistical MT system that translates (say) French sentences into English, is divided into three parts: (1) a language model (LM) that assigns a probability $\mathrm{P}(\mathrm{e})$ to any English string, (2) a translation model (TM) that assigns a probability $\mathrm{P}(\mathrm{f} \mid \mathrm{e})$ to any pair of English and French strings, and (3) a decoder. The decoder takes a previously unseen sentence $f$ and tries to find the $e$ that maximizes $\mathrm{P}(\mathrm{e} \mid \mathrm{f})$, or equivalently maximizes $\mathrm{P}(\mathrm{e}) \cdot \mathrm{P}(\mathrm{f} \mid \mathrm{e})$.

Brown et al. (1993) introduced a series of TMs based on word-for-word substitution and reordering, but did not include a decoding algorithm. If the source and target languages are constrained to have the same word order (by choice or through suitable pre-processing), then the linear Viterbi algorithm can be applied (Tillmann et al., 1997). If re-ordering is limited to rotations around nodes in a binary tree, then optimal decoding can be carried out by a high-polynomial algorithm (Wu, 1996). For arbitrary word-reordering, the decoding problem is NP-complete (Knight, 1999).

A sensible strategy (Brown et al., 1995; Wang and Waibel, 1997) is to examine a large subset of likely decodings and choose just from that. Of course, it is possible to miss a good translation this way. If the decoder returns $\mathrm{e}^{\prime}$ but there exists some e for which $\mathrm{P}(\mathrm{e} \mid \mathrm{f})>\mathrm{P}\left(\mathrm{e}^{\prime} \mid \mathrm{f}\right)$, this is called a search error. As Wang and Waibel (1997) remark, it is hard to know whether a search error has occurred-the only way to show that a decoding is sub-optimal is to actually produce a higherscoring one.

Thus, while decoding is a clear-cut optimization task in which every problem instance has a right answer, it is hard to come up with good answers quickly. This paper reports on measurements of speed, search errors, and translation quality in the context of a traditional stack decoder (Jelinek, 1969; Brown et al., 1995) and two new decoders. The first is a fast greedy decoder, and the second is a slow optimal decoder based on generic mathematical programming techniques.

\section{IBM Model 4}

In this paper, we work with IBM Model 4, which revolves around the notion of a word alignment over a pair of sentences (see Figure 1). A word alignment assigns a single home (English string position) to each French word. If two French words align to the same English word, then that 


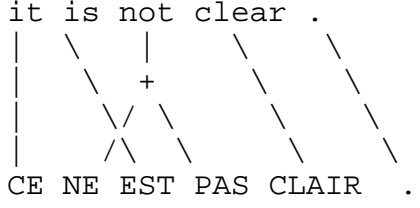

Figure 1: Sample word alignment.

English word is said to have a fertility of two. Likewise, if an English word remains unalignedto, then it has fertility zero. The word alignment in Figure 1 is shorthand for a hypothetical stochastic process by which an English string gets converted into a French string. There are several sets of decisions to be made.

First, every English word is assigned a fertility. These assignments are made stochastically according to a table $\mathrm{n}\left(\phi \mid \mathrm{e}_{i}\right)$. We delete from the string any word with fertility zero, we duplicate any word with fertility two, etc. If a word has fertility greater than zero, we call it fertile. If its fertility is greater than one, we call it very fertile.

After each English word in the new string, we may increment the fertility of an invisible English NULL element with probability $\mathrm{p}_{1}$ (typically about 0.02 ). The NULL element ultimately produces "spurious" French words.

Next, we perform a word-for-word replacement of English words (including NULL) by French words, according to the table $\mathrm{t}\left(\mathrm{f}_{j} \mid \mathrm{e}_{i}\right)$.

Finally, we permute the French words. In permuting, Model 4 distinguishes between French words that are heads (the leftmost French word generated from a particular English word), nonheads (non-leftmost, generated only by very fertile English words), and NULL-generated.

Heads. The head of one English word is assigned a French string position based on the position assigned to the previous English word. If an English word $\mathrm{e}_{i-1}$ translates into something at French position j, then the French head word of $\mathrm{e}_{i}$ is stochastically placed in French position $\mathrm{k}$ with distortion probability $\mathrm{d}_{1}\left(\mathrm{k}-\mathrm{j} \mid \operatorname{class}\left(\mathrm{e}_{i-1}\right)\right.$, class $\left(\mathrm{f}_{k}\right)$ ), where "class" refers to automatically determined word classes for French and English vocabulary items. This relative offset $\mathrm{k}-\mathrm{j}$ encourages adjacent English words to translate into adjacent French words. If $\mathrm{e}_{i-1}$ is infertile, then $\mathrm{j}$ is taken from $\mathrm{e}_{i-2}$, etc. If $\mathrm{e}_{i-1}$ is very fertile, then $\mathrm{j}$ is the average of the positions of its French translations.

Non-heads. If the head of English word $\mathrm{e}_{i}$ is placed in French position $\mathrm{j}$, then its first nonhead is placed in French position $\mathrm{k}(>\mathrm{j})$ according to another table $\mathrm{d}_{>1}\left(\mathrm{k}-\mathrm{j} \mid \operatorname{class}\left(\mathrm{f}_{k}\right)\right)$. The next non-head is placed at position $\mathrm{q}$ with probability $\mathrm{d}_{>1}\left(\mathrm{q}-\mathrm{k} \mid \operatorname{class}\left(\mathrm{f}_{q}\right)\right)$, and so forth.

NULL-generated. After heads and non-heads are placed, NULL-generated words are permuted into the remaining vacant slots randomly. If there are $\phi_{0}$ NULL-generated words, then any placement scheme is chosen with probability $1 / \phi_{0}$ !.

These stochastic decisions, starting with e, result in different choices of $f$ and an alignment of $f$ with e. We map an e onto a particular $<$ a,f $>$ pair with probability:

$$
\begin{aligned}
& \mathrm{P}(\mathrm{a}, \mathrm{f} \mid \mathrm{e})= \\
& \prod_{i=1}^{l} \mathrm{n}\left(\phi_{i} \mid \mathrm{e}_{i}\right) \times \prod_{i=1}^{l} \prod_{k=1}^{\phi_{i}} \mathrm{t}\left(\tau_{i k} \mid \mathrm{e}_{i}\right) \times \\
& \quad \prod_{i=1, \phi_{i}>0}^{l} \mathrm{~d}_{1}\left(\pi_{i 1}-c_{\rho_{i}} \mid \operatorname{class}\left(\mathrm{e}_{\rho_{i}}\right), \operatorname{class}\left(\tau_{i 1}\right)\right) \times \\
& \prod_{i=1}^{l} \prod_{k=2}^{\phi_{i}} \mathrm{~d}_{>1}\left(\pi_{i k}-\pi_{i(k-1)} \mid \operatorname{class}\left(\tau_{i k}\right)\right) \times \\
& \left(\begin{array}{c}
m-\phi_{0} \\
\phi_{0}
\end{array}\right) p_{1}{ }^{\phi_{0}}\left(1-p_{1}\right)^{m-2 \phi_{0}} \times \\
& \prod_{k=1}^{\phi_{0}} t\left(\tau_{0 k} \mid \mathrm{NULL}\right)
\end{aligned}
$$

where the factors separated by $\times$ symbols denote fertility, translation, head permutation, non-head permutation, null-fertility, and null-translation probabilities. $^{1}$

\section{Definition of the Problem}

If we observe a new sentence $f$, then an optimal decoder will search for an e that maximizes $\mathrm{P}(\mathrm{e} \mid \mathrm{f})$

\footnotetext{
${ }^{1}$ The symbols in this formula are: $l$ (the length of e), $m$ (the length of $\mathrm{f}$ ), $\mathrm{e}_{i}$ (the $\mathrm{i}^{\text {th }}$ English word in e), $\mathrm{e}_{0}$ (the NULL word), $\phi_{i}$ (the fertility of $\mathrm{e}_{i}$ ), $\phi_{0}$ (the fertility of the NULL word), $\tau_{i k}$ (the $\mathrm{k}^{t h}$ French word produced by $\mathrm{e}_{i}$ in a), $\pi_{i k}$ (the position of $\tau_{i k}$ in f), $\rho_{i}$ (the position of the first fertile word to the left of $\mathrm{e}_{i}$ in a), $c_{\rho_{i}}$ (the ceiling of the average of all $\pi_{\rho_{i} k}$ for $\rho_{i}$, or 0 if $\rho_{i}$ is undefined).
} 
$\simeq \mathrm{P}(\mathrm{e}) \cdot \mathrm{P}(\mathrm{f} \mid \mathrm{e})$. Here, $\mathrm{P}(\mathrm{f} \mid \mathrm{e})$ is the sum of $\mathrm{P}(\mathrm{a}, \mathrm{f} \mid \mathrm{e})$ over all possible alignments a. Because this sum involves significant computation, we typically avoid it by instead searching for an $\langle\mathrm{e}, \mathrm{a}\rangle$ pair that maximizes $\mathrm{P}(\mathrm{e}, \mathrm{a} \mid \mathrm{f}) \simeq \mathrm{P}(\mathrm{e}) \cdot \mathrm{P}(\mathrm{a}, \mathrm{f} \mid \mathrm{e})$. We take the language model $\mathrm{P}(\mathrm{e})$ to be a smoothed n-gram model of English.

\section{Stack-Based Decoding}

The stack (also called $\mathrm{A}^{*}$ ) decoding algorithm is a kind of best-first search which was first introduced in the domain of speech recognition (Jelinek, 1969). By building solutions incrementally and storing partial solutions, or hypotheses, in a "stack" (in modern terminology, a priority queue), the decoder conducts an ordered search of the solution space. In the ideal case (unlimited stack size and exhaustive search time), a stack decoder is guaranteed to find an optimal solution; our hope is to do almost as well under real-world constraints of limited space and time. The generic stack decoding algorithm follows:

- Initialize the stack with an empty hypothesis.

- Pop $h$, the best hypothesis, off the stack.

- If $h$ is a complete sentence, output $h$ and terminate.

- For each possible next word $w$, extend $h$ by adding $w$ and push the resulting hypothesis onto the stack.

- Return to the second step (pop).

One crucial difference between the decoding process in speech recognition (SR) and machine translation (MT) is that speech is always produced in the same order as its transcription. Consequently, in SR decoding there is always a simple left-to-right correspondence between input and output sequences. By contrast, in MT the leftto-right relation rarely holds even for language pairs as similar as French and English. We address this problem by building the solution from left to right, but allowing the decoder to consume its input in any order. This change makes decoding significantly more complex in MT; instead of knowing the order of the input in advance, we must consider all $n$ ! permutations of an $n$-word input sentence.

Another important difference between SR and MT decoding is the lack of reliable heuristics in MT. A heuristic is used in A* search to estimate the cost of completing a partial hypothesis. A good heuristic makes it possible to accurately compare the value of different partial hypotheses, and thus to focus the search in the most promising direction. The left-to-right restriction in SR makes it possible to use a simple yet reliable class of heuristics which estimate cost based on the amount of input left to decode. Partly because of the absence of left-to-right correspondence, MT heuristics are significantly more difficult to develop (Wang and Waibel, 1997). Without a heuristic, a classic stack decoder is ineffective because shorter hypotheses will almost always look more attractive than longer ones, since as we add words to a hypothesis, we end up multiplying more and more terms to find the probability. Because of this, longer hypotheses will be pushed off the end of the stack by shorter ones even if they are in reality better decodings. Fortunately, by using more than one stack, we can eliminate this effect.

In a multistack decoder, we employ more than one stack to force hypotheses to compete fairly. More specifically, we have one stack for each subset of input words. This way, a hypothesis can only be pruned if there are other, better, hypotheses that represent the same portion of the input. With more than one stack, however, how does a multistack decoder choose which hypothesis to extend during each iteration? We address this issue by simply taking one hypothesis from each stack, but a better solution would be to somehow compare hypotheses from different stacks and extend only the best ones.

The multistack decoder we describe is closely patterned on the Model 3 decoder described in the (Brown et al., 1995) patent. We build solutions incrementally by applying operations to hypotheses. There are four operations:

- Add adds a new English word and aligns a single French word to it.

- AddZfert adds two new English words. The first has fertility zero, while the second is aligned to a single French word.

- Extend aligns an additional French word to the most recent English word, increasing its fertility. 
- AddNull aligns a French word to the English NULL element.

AddZfert is by far the most expensive operation, as we must consider inserting a zero-fertility English word before each translation of each unaligned French word. With an English vocabulary size of 40,000, AddZfert is 400,000 times more expensive than AddNull!

We can reduce the cost of AddZfert in two ways. First, we can consider only certain English words as candidates for zero-fertility, namely words which both occur frequently and have a high probability of being assigned frequency zero. Second, we can only insert a zero-fertility word if it will increase the probability of a hypothesis. According to the definition of the decoding problem, a zero-fertility English word can only make a decoding more likely by increasing $\mathrm{P}(\mathrm{e})$ more than it decreases $\mathrm{P}(\mathrm{a}, \mathrm{f} \mid \mathrm{e}) .^{2}$ By only considering helpful zero-fertility insertions, we save ourselves significant overhead in the AddZfert operation, in many cases eliminating all possibilities and reducing its cost to less than that of AddNull.

\section{Greedy Decoding}

Over the last decade, many instances of NPcomplete problems have been shown to be solvable in reasonable/polynomial time using greedy methods (Selman et al., 1992; Monasson et al., 1999). Instead of deeply probing the search space, such greedy methods typically start out with a random, approximate solution and then try to improve it incrementally until a satisfactory solution is reached. In many cases, greedy methods quickly yield surprisingly good solutions.

We conjectured that such greedy methods may prove to be helpful in the context of MT decoding. The greedy decoder that we describe starts the translation process from an English gloss of the French sentence given as input. The gloss is constructed by aligning each French word $\mathrm{f}_{j}$ with its most likely English translation $\mathrm{e}_{\mathrm{f}_{j}}\left(\mathrm{e}_{\mathrm{f}_{j}}=\right.$ $\left.\operatorname{argmax}_{e} \mathrm{t}\left(\mathrm{e} \mid \mathrm{f}_{j}\right)\right)$. For example, in translating the French sentence "Bien entendu, il parle de une belle victoire .", the greedy decoder initially as-

\footnotetext{
${ }^{2}$ We know that adding a zero-fertility word will decrease $\mathrm{P}(\mathrm{a}, \mathrm{f} \mid \mathrm{e})$ because it adds a term $\mathrm{n}\left(0 \mid \mathrm{e}_{i}\right)<1$ to the calculation.
}

sumes that a good translation of it is "Well heard , it talking a beautiful victory" because the best translation of "bien" is "well", the best translation of "entendu" is "heard", and so on. The alignment corresponding to this translation is shown at the top of Figure 2.

Once the initial alignment is created, the greedy decoder tries to improve it, i.e., tries to find an alignment (and implicitly translation) of higher probability, by applying one of the following operations:

- translateOneOrTwoWords $\left(j_{1}, \mathrm{e}_{1}, j_{2}, \mathrm{e}_{2}\right)$ changes the translation of one or two French words, those located at positions $j_{1}$ and $j_{2}$, from $\mathrm{e}_{f_{j_{1}}}$ and $\mathrm{e}_{f_{j_{2}}}$ into $\mathrm{e}_{1}$ and $\mathrm{e}_{2}$. If $\mathrm{e}_{f_{j}}$ is a word of fertility 1 and $\mathrm{e}_{k}$ is NULL, then $\mathrm{e}_{f_{j}}$ is deleted from the translation. If $\mathrm{e}_{f_{j}}$ is the NULL word, the word $\mathrm{e}_{k}$ is inserted into the translation at the position that yields the alignment of highest probability. If $\mathrm{e}_{j_{j_{1}}}=$ $\mathrm{e}_{1}$ or $\mathrm{e}_{f_{2}}=\mathrm{e}_{2}$, this operation amounts to changing the translation of a single word.

- translateAndInsert $\left(j, e_{1}, e_{2}\right)$ changes the translation of the French word located at position $j$ from $\mathrm{e}_{f_{j}}$ into $e_{1}$ and simulataneously inserts word $\mathrm{e}_{2}$ at the position that yields the alignment of highest probability. Word $e_{2}$ is selected from an automatically derived list of 1024 words with high probability of having fertility 0 . When $\mathrm{e}_{f_{j}}=\mathrm{e}_{1}$, this operation amounts to inserting a word of fertility 0 into the alignment.

- removeWordOfFertility0 $(i)$ deletes the word of fertility 0 at position $i$ in the current alignment.

- swapSegments $\left(i_{1}, i_{2}, j_{1}, j_{2}\right)$ creates a new alignment from the old one by swapping non-overlapping English word segments $\left[i_{1}, i_{2}\right]$ and $\left[j_{1}, j_{2}\right]$. During the swap operation, all existing links between English and French words are preserved. The segments can be as small as a word or as long as $|e|-1$ words, where $|e|$ is the length of the English sentence.

- joinWords $\left(i_{1}, i_{2}\right)$ eliminates from the alignment the English word at position $i_{1}$ (or $i_{2}$ ) and links the French words generated by $e_{i_{1}}$ (or $e_{i_{2}}$ ) to $e_{i_{2}}\left(\right.$ or $\left.e_{i_{1}}\right)$. 


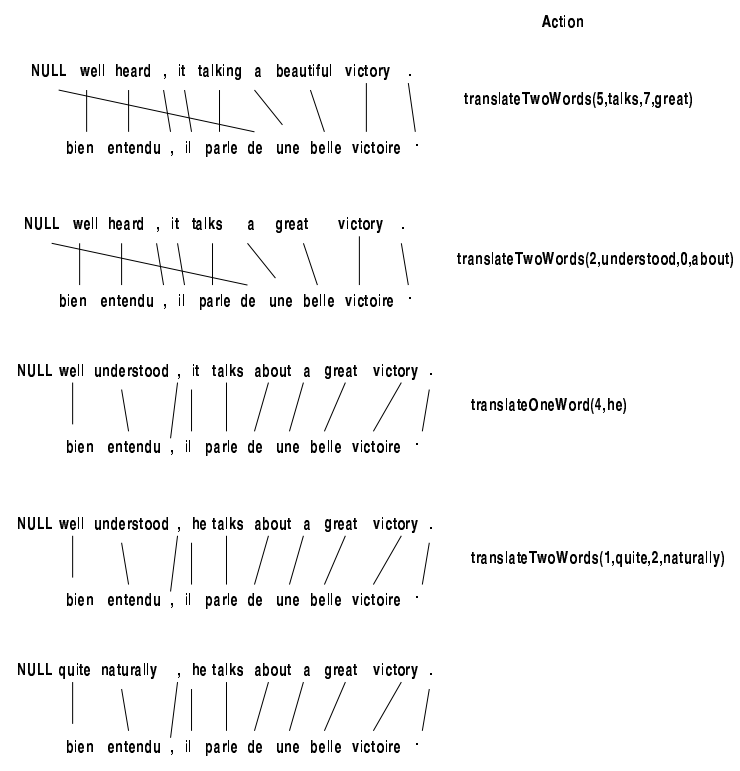

Figure 2: Example of how the greedy decoder produces the translation of French sentence "Bien entendu, il parle de une belle victoire."

In a stepwise fashion, starting from the initial gloss, the greedy decoder iterates exhaustively over all alignments that are one operation away from the alignment under consideration. At every step, the decoder chooses the alignment of highest probability, until the probability of the current alignment can no longer be improved. When it starts from the gloss of the French sentence "Bien entendu, il parle de une belle victoire.", for example, the greedy decoder alters the initial alignment incrementally as shown in Figure 2, eventually producing the translation "Quite naturally, he talks about a great victory.". In the process, the decoder explores a total of 77421 distinct alignments/translations, of which "Quite naturally, he talks about a great victory." has the highest probability.

We chose the operation types enumerated above for two reasons: (i) they are general enough to enable the decoder escape local maxima and modify in a non-trivial manner a given alignment in order to produce good translations; (ii) they are relatively inexpensive (timewise). The most time consuming operations in the decoder are swapSegments, translateOneOrTwoWords, and translateAndInsert. SwapSegments iterates over all possible non-overlapping span pairs that can be built on a sequence of length $|e|$.
TranslateOneOrTwoWords iterates over $|f|^{2}$ $\times|t|^{2}$ alignments, where $|f|$ is the size of the French sentence and $|t|$ is the number of translations we associate with each word (in our implementation, we limit this number to the top 10 translations). TranslateAndInsert iterates over $|f| \times|t| \times|z|$ alignments, where $|z|$ is the size of the list of words with high probability of having fertility 0 (1024 words in our implementation).

\section{Integer Programming Decoding}

Knight (1999) likens MT decoding to finding optimal tours in the Traveling Salesman Problem (Garey and Johnson, 1979)—choosing a good word order for decoder output is similar to choosing a good TSP tour. Because any TSP problem instance can be transformed into a decoding problem instance, Model 4 decoding is provably NP-complete in the length of $\mathrm{f}$. It is interesting to consider the reverse direction-is it possible to transform a decoding problem instance into a TSP instance? If so, we may take great advantage of previous research into efficient TSP algorithms. We may also take advantage of existing software packages, obtaining a sophisticated decoder with little programming effort.

It is difficult to convert decoding into straight TSP, but a wide range of combinatorial optimization problems (including TSP) can be expressed in the more general framework of linear integer programming. A sample integer program (IP) looks like this:

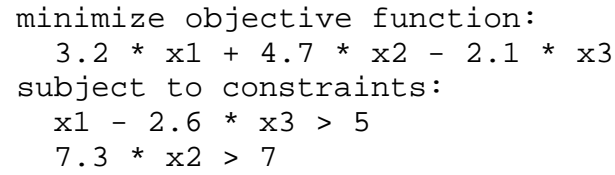

A solution to an IP is an assignment of integer values to variables. Solutions are constrained by inequalities involving linear combinations of variables. An optimal solution is one that respects the constraints and minimizes the value of the objective function, which is also a linear combination of variables. We can solve IP instances with generic problem-solving software such as Ip_solve or CPLEX. ${ }^{3}$ In this section we explain

\footnotetext{
${ }^{3}$ Available at ftp://ftp.ics.ele.tue.nl/pub/lp_solve and
} http://www.cplex.com. 


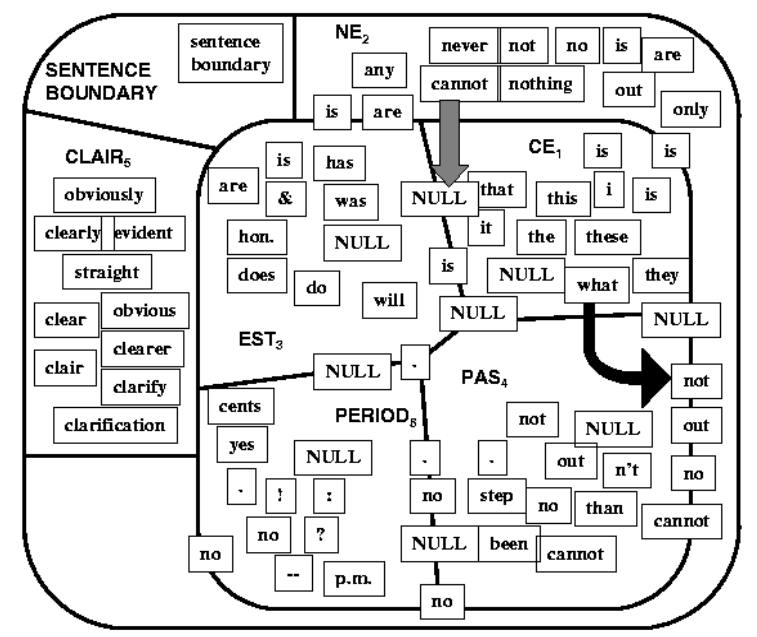

Figure 3: A salesman graph for the input sentence $\mathrm{f}=$ "CE NE EST PAS CLAIR ." There is one city for each word in $\mathrm{f}$. City boundaries are marked with bold lines, and hotels are illustrated with rectangles. A tour of cities is a sequence of hotels (starting at the sentence boundary hotel) that visits each city exactly once before returning to the start.

how to express MT decoding (Model 4 plus English bigrams) in IP format.

We first create a salesman graph like the one in Figure 3. To do this, we set up a city for each word in the observed sentence f. City boundaries are shown with bold lines. We populate each city with ten hotels corresponding to ten likely English word translations. Hotels are shown as small rectangles. The owner of a hotel is the English word inside the rectangle. If two cities have hotels with the same owner $\mathrm{x}$, then we build a third $\mathrm{x}$ owned hotel on the border of the two cities. More generally, if $n$ cities all have hotels owned by $\mathrm{x}$, we build $2^{n}-n-1$ new hotels (one for each non-empty, non-singleton subset of the cities) on various city borders and intersections. Finally, we add an extra city representing the sentence boundary.

We define a tour of cities as a sequence and hotels (starting at the sentence boundary hotel) that visits each city exactly once before returning to the start. If a hotel sits on the border between two cities, then staying at that hotel counts as visiting both cities. We can view each tour of cities as corresponding to a potential decoding $<\mathrm{e}, \mathrm{a}\rangle$.
The owners of the hotels on the tour give us e, while the hotel locations yield a.

The next task is to establish real-valued (asymmetric) distances between pairs of hotels, such that the length of any tour is exactly the negative of $\log (\mathrm{P}(\mathrm{e}) \cdot \mathrm{P}(\mathrm{a}, \mathrm{f} \mid \mathrm{e}))$. Because $\log$ is monotonic, the shortest tour will correspond to the likeliest decoding.

The distance we assign to each pair of hotels consists of some small piece of the Model 4 formula. The usual case is typified by the large black arrow in Figure 3. Because the destination hotel "not" sits on the border between cities NE and PAS, it corresponds to a partial alignment in which the word "not" has fertility two:

$$
\begin{gathered}
\text {... what not } \ldots \\
\mathrm{CE} \text { NE EST PAS CLAIR. }
\end{gathered}
$$

If we assume that we have already paid the price for visiting the "what" hotel, then our interhotel distance need only account for the partial alignment concerning "not":

$$
\begin{aligned}
& \text { distance }= \\
& \quad-\log (\text { bigram }(\text { not } \mid \text { what })) \\
& -\log (\mathrm{n}(2 \mid \text { not })) \\
& -\log (\mathrm{t}(\mathrm{NE} \mid \text { not }))-\log (\mathrm{t}(\mathrm{PAS} \mid \text { not })) \\
& -\log \left(\mathrm{d}_{1}(+1 \mid \text { class }(\text { what }), \text { class }(\mathrm{NE}))\right) \\
& \left.-\log \left(\mathrm{d}_{>1}(+2 \mid \text { class(PAS })\right)\right)
\end{aligned}
$$

NULL-owned hotels are treated specially. We require that all non-NULL hotels be visited before any NULL hotels, and we further require that at most one NULL hotel visited on a tour. Moreover, the NULL fertility sub-formula is easy to compute if we allow only one NULL hotel to be visited: $\phi_{0}$ is simply the number of cities that hotel straddles, and $m$ is the number of cities minus one. This case is typified by the large gray arrow shown in Figure 3.

Between hotels that are located (even partially) in the same city, we assign an infinite distance in both directions, as travel from one to the other can never be part of a tour. For 6-word French sentences, we normally come up with a graph that has about 80 hotels and 3500 finite-cost travel segments.

The next step is to cast tour selection as an integer program. Here we adapt a subtour elimination strategy used in standard TSP. We create a binary $(0 / 1)$ integer variable $x_{i j}$ for each pair of hotels $i$ 
and $j . x_{i j}=1$ if and only if travel from hotel $i$ to hotel $j$ is on the itinerary. The objective function is straightforward:

$$
\text { minimize: } \sum_{(i, j)} x_{i j} \cdot \operatorname{distance}(i, j)
$$

This minimization is subject to three classes of constraints. First, every city must be visited exactly once. That means exactly one tour segment must exit each city:

$$
\forall_{c \in \text { cities }}: \sum_{\substack{i \text { located at least } \\ \text { partially in } c}} \sum_{j} x_{i j}=1
$$

Second, the segments must be linked to one another, i.e., every hotel has either (a) one tour segment coming in and one going out, or (b) no segments in and none out. To put it another way, every hotel must have an equal number of tour segments going in and out:

$$
\forall_{i}: \sum_{j} x_{i j}=\sum_{j} x_{j i}
$$

Third, it is necessary to prevent multiple independent sub-tours. To do this, we require that every proper subset of cities have at least one tour segment leaving it:

$$
\forall_{s \subset \text { cities }}: \sum_{\substack{i \text { located } \\ \text { entirely } \\ \text { within } s}} \sum_{\substack{j \text { located } \\ \text { at least } \\ \text { partially } \\ \text { outside } s}} x_{i j}>=1
$$

There are an exponential number of constraints in this third class.

Finally, we invoke our IP solver. If we assign mnemonic names to the variables, we can easily extract $\langle$ e,a $\rangle$ from the list of variables and their binary values. The shortest tour for the graph in Figure 3 corresponds to this optimal decoding: it is not clear.

We can obtain the second-best decoding by adding a new constraint to the IP to stop it from choosing the same solution again. ${ }^{4}$

\footnotetext{
${ }^{4}$ If we simply replace "minimize" with "maximize," we can obtain the longest tour, which corresponds to the worst decoding!
}

\section{Experiments and Discussion}

In our experiments we used a test collection of 505 sentences, uniformly distributed across the lengths $6,8,10,15$, and 20 . We evaluated all decoders with respect to (1) speed, (2) search optimality, and (3) translation accuracy. The last two factors may not always coincide, as Model 4 is an imperfect model of the translation process-i.e., there is no guarantee that a numerically optimal decoding is actually a good translation.

Suppose a decoder outputs $e^{\prime}$, while the optimal decoding turns out to be $e$. Then we consider six possible outcomes:

- no error (NE): $e^{\prime}=e$, and $e^{\prime}$ is a perfect translation.

- pure model error (PME): $e^{\prime}=e$, but $e^{\prime}$ is not a perfect translation.

- deadly search error (DSE): $e^{\prime} \neq e$, and while $e$ is a perfect translation, while $e^{\prime}$ is not.

- fortuitous search error (FSE): $e^{\prime} \neq e$, and $e^{\prime}$ is a perfect translation, while $e$ is not.

- harmless search error (HSE): $e^{\prime} \neq e$, but $e^{\prime}$ and $e$ are both perfectly good translations.

- compound error (CE): $e^{\prime} \neq e$, and neither is a perfect translation.

Here, "perfect" refers to a human-judged translation that transmits all of the meaning of the source sentence using flawless target-language syntax.

We have found it very useful to have several decoders on hand. It is only through IP decoder output, for example, that we can know the stack decoder is returning optimal solutions for so many sentences (see Table 1). The IP and stack decoders enabled us to quickly locate bugs in the greedy decoder, and to implement extensions to the basic greedy search that can find better solutions. (We came up with the greedy operations discussed in Section 5 by carefully analyzing error logs of the kind shown in Table 1). The results in Table 1 also enable us to prioritize the items on our research agenda. Since the majority of the translation errors can be attributed to the language and translation models we use (see column PME in Table 1), it is clear that significant improvement in translation quality will come from better 


\begin{tabular}{|c|c|c|c|c|c|c|c|c|c|c|}
\hline $\begin{array}{l}\text { sent } \\
\text { length }\end{array}$ & $\begin{array}{l}\text { decoder } \\
\text { type }\end{array}$ & $\begin{array}{r}\text { time } \\
\text { (sec/sent) }\end{array}$ & $\begin{array}{r}\text { search } \\
\text { errors }\end{array}$ & $\begin{array}{r}\text { translation } \\
\text { errors (semantic } \\
\text { and/or syntactic) }\end{array}$ & $\mathrm{NE}$ & PME & DSE & FSE & HSE & $\mathrm{CE}$ \\
\hline 6 & IP & 47.50 & 0 & 57 & 44 & 57 & 0 & 0 & 0 & 0 \\
\hline 6 & stack & 0.79 & 5 & 58 & 43 & 53 & 1 & 0 & 0 & 4 \\
\hline 6 & greedy & 0.07 & 18 & 60 & 38 & 45 & 5 & 2 & 1 & 10 \\
\hline 8 & IP & 499.00 & 0 & 76 & 27 & 74 & 0 & 0 & 0 & 0 \\
\hline 8 & stack & 5.67 & 20 & 75 & 24 & 57 & 1 & 2 & 2 & 15 \\
\hline 8 & greedy & 2.66 & 43 & 75 & 20 & 38 & 4 & 5 & 1 & 33 \\
\hline
\end{tabular}

Table 1: Comparison of decoders on sets of 101 test sentences. All experiments in this table use a bigram language model.

\begin{tabular}{|ll|r|r|}
\hline $\begin{array}{l}\text { Sent } \\
\text { length }\end{array}$ & $\begin{array}{l}\text { decoder } \\
\text { type }\end{array}$ & $\begin{array}{r}\text { time } \\
\text { (sec/sent) }\end{array}$ & $\begin{array}{r}\text { translation } \\
\text { errors (semantic } \\
\text { and/or syntactic) }\end{array}$ \\
\hline 6 & stack & 13.72 & 42 \\
6 & greedy & 1.58 & 46 \\
6 & greedy & 0.07 & 46 \\
\hline 8 & stack & 45.45 & 59 \\
8 & greedy & 2.75 & 68 \\
8 & greedy & 0.15 & 69 \\
\hline 10 & stack & 105.15 & 57 \\
10 & greedy & 3.83 & 63 \\
10 & greedy & 0.20 & 68 \\
\hline 15 & stack & $>2000$ & 74 \\
15 & greedy $^{*}$ & 12.06 & 75 \\
15 & greedy $^{*}$ & 1.11 & 75 \\
15 & greedy $^{1}$ & 0.63 & 76 \\
\hline 20 & greedy & 49.23 & 86 \\
20 & greedy & 11.34 & 93 \\
20 & greedy & & 93 \\
\hline
\end{tabular}

algorithm. When the threshold is set to one second per sentence (the greedy ${ }^{1}$ label in Table 1), the performance is affected only slightly.

Acknowledgments. This work was supported by DARPA-ITO grant N66001-00-1-9814.

\section{References}

P. Brown, S. Della Pietra, V. Della Pietra, and R. Mercer. 1993. The mathematics of statistical machine translation: Parameter estimation. Computational Linguistics, 19(2).

P. Brown, J. Cocke, S. Della Pietra, V. Della Pietra, F. Jelinek, J. Lai, and R. Mercer. 1995. Method and system for natural language translation. U.S. Patent 5,477,451.

Table 2: Comparison between decoders using a trigram language model. Greedy* and greedy ${ }^{1}$ are greedy decoders optimized for speed.

models.

The results in Table 2, obtained with decoders that use a trigram language model, show that our greedy decoding algorithm is a viable alternative to the traditional stack decoding algorithm. Even when the greedy decoder uses an optimized-forspeed set of operations in which at most one word is translated, moved, or inserted at a time and at most 3-word-long segments are swapped-which is labeled "greedy" in Table 2-the translation accuracy is affected only slightly. In contrast, the translation speed increases with at least one order of magnitude. Depending on the application of interest, one may choose to use a slow decoder that provides optimal results or a fast, greedy decoder that provides non-optimal, but acceptable results. One may also run the greedy decoder using a time threshold, as any instance of anytime

M. Garey and D. Johnson. 1979. Computers and Intractability. A Guide to the Theory of NPCompleteness. W.H. Freeman and Co., New York.

F. Jelinek. 1969. A fast sequential decoding algorithm using a stack. IBM Research Journal of Research and Development, 13.

K. Knight. 1999. Decoding complexity in wordreplacement translation models. Computational Linguistics, 25(4).

R. Monasson, R. Zecchina, S. Kirkpatrick, B. Selman, and L. Troyansky. 1999. Determining computational complexity from characteristic "phase transitions'. Nature, 800(8).

B. Selman, H. Levesque, and D. Mitchell. 1992. A new method for solving hard satisfiability problems. In Proc. AAAI.

C. Tillmann, S. Vogel, H. Ney, and A. Zubiaga. 1997. A DP-based search using monotone alignments in statistical translation. In Proc. ACL.

Y. Wang and A. Waibel. 1997. Decoding algorithm in statistical machine translation. In Proc. ACL.

D. Wu. 1996. A polynomial-time algorithm for statistical machine translation. In Proc. ACL. 